\title{
Tau accumulation in the nucleus accumbens in tangle-predominant dementia
}

\author{
Ito Kawakamii ${ }^{1,2}$, Masato Hasegawa ${ }^{3}$, Tetsuaki Arai ${ }^{1,4}$, Kenji Ikeda $^{1,5}$, Kenichi Oshima ${ }^{6}$, Kazuhiro Niizato ${ }^{6}$, \\ Naoya Aoki ${ }^{1,2}$, Katsuse Omi ${ }^{2}$, Shinji Higashi ${ }^{1}$, Masato Hosokawa ${ }^{1}$, Yoshio Hirayasu ${ }^{2}$ and Haruhiko Akiyama ${ }^{1 *}$
}

\begin{abstract}
Background: Tangle-predominant dementia (TPD) is characterized neuropathologically by numerous neurofibrillary tangles in the limbic areas with no or occasional senile plaques throughout the brain. TPD is an under-recognized disease, while it is a common cause of dementia in those over 80 years of age. In the present study, we describe hyperphosphorylated tau (tau) accumulation in the nucleus accumbens (Acb) in patients with TPD.

Results: We investigated immunohistochemically the brain tissues from 7 patients with TPD, 22 with Alzheimer disease (AD) and 11 non-demented aged subjects. In the Acb of all 7 TPD patients, a considerable number of tau positive neurons were found together with many neuropil threads. The tau deposits in the Acb were labeled with all the anti-tau antibodies used in the present study. They included conformational change-specific, phosphorylation-specific and phosphorylation-independent antibodies. The Acb consists of the predominant medium-sized neurons with a small number of large neurons. Both the cell types were affected by tau pathology in TPD. Tau accumulation in the majority of such neurons appeared to be pretangle-like, diffuse deposits with only occasional paired helical filament formation. Tau positive neurons were also found in the Acb in some AD and non-demented aged subjects but much fewer in the majority of cases. The immunoblot analyses of fresh frozen samples of the Acb and parahippocampal cortex from 3 TPD and 3 AD patients revealed that the insoluble tau in the Acb was a mixture of the 3- and 4-repeat isoforms.

Conclusions: To our knowledge, this is the first report on the occurrence of tau accumulation in the Acb in TPD. The Acb receives direct and massive projections from the hippocampal CA1 and subiculum where neurofibrillary tangles are known to occur more frequently in TPD than in AD. The prevalence of abnormal tau accumulation in the Acb in TPD may support the idea that abnormal tau aggregation propagates via neural circuits. In all but one TPD cases used in this study, delusion was a consistent clinical feature. Whether the Acb tau accumulation is related to the psychiatric symptoms in TPD may be an issue for further investigation.
\end{abstract}

Keywords: Neurofibrillary tangle, Alzheimer disease, Propagation, Delusion

\section{Introduction}

Tangle-predominant dementia (TPD), which is also referred to as neurofibrillary tangle predominant dementia, limbic neurofibrillary tangle dementia or senile dementia of the neurofibrillary tangle type, is a poorly understood and under-recognized tauopathy. TPD has been reported to comprise 0.7 to $5.8 \%$ of elderly patients with dementia [1-3]. TPD is characterized neuropathologically by numerous neurofibrillary tangles (NFT) in the limbic areas with

\footnotetext{
* Correspondence: akiyama-hr@igakuken.or.jp

'Dementia Research Project, Tokyo Metropolitan Institute of Medical Science, 2-1-6 Kamikitazawa, Setagaya-ku, Tokyo 156-8506, Japan

Full list of author information is available at the end of the article
}

no or occasional senile plaques throughout the brain. The clinical features of TPD include the late-adult onset, which is over 80 years in the majority of cases, and slow progression of dementia as compared with Alzheimer's disease (AD). In patients with TPD, there is a propensity for the memory disturbance to be conspicuous with relative preservation of other cognitive functions. However, it is hard to distinguish TPD from $\mathrm{AD}$ on a clinical basis and, thus, diagnosis of TPD in most cases is only made postmortem.

The etiology of TPD is unknown. NFT in TPD consist of both 3-repeat (3R) and 4-repeat (4R) isoforms of hyperphosphorylated tau (tau), and the neuronal cell types 
bearing NFT in TPD are similar to those in AD. TPD seems to be a disorder that is related to $\mathrm{AD}$, if it is not an atypical form of AD. TPD, as a subtype of tauopathy, is also included in the group described as neuropathologicallydefined frontotemporal lobar degeneration [4,5]. In fact, cortical lesions in TPD are localized to the mediobasal temporal cortex. Thus, the situation of TPD in the groups of dementing neurodegenerative diseases remains unclear from both clinical and neuropathological points of view.

A neuropathological characteristic of TPD is the heavy accumulation of NFT in the hippocampal regions, with few or occasional NFT in neocortical areas beyond the collateral sulcus. Compared with AD patients in which a similar number of NFT occurs in the hippocampal regions, neuronal cell loss, tissue rarefaction and gliosis are less prominent in TPD, even in NFT rich areas. Changes in the neocortex are modest, with wellpreserved laminar structures and unremarkable neuronal cell loss. The cortical expansion of NFT in TPD is considered to follow in principle the hierarchical pathway described in AD by Braak and Braak [6] but to be limited to stage IV. In the hippocampal regions, the density of NFT is higher than in AD [7] and ghost tangles are very frequent [3]. Tau pathology in the subcortical structures in TPD has not been well studied. The occurrence of NFT in the amygdala, the nucleus basalis of Meynert, the substantia nigra and the locus coeruleus, regions where NFT frequently occur in $\mathrm{AD}$ cases, have been reported in TPD $[3,8]$.

The nucleus accumbens (Acb) is located in the region where the caudate head and the rostral putamen meet near the septum pellucidum (Figure 1). The Acb and the olfactory tubercle form the ventral striatum in the forebrain. The Acb is a key component of the limbic striatal loop in which the Acb receives fibers from the prefrontal cortex, amygdala, hippocampus and ventral tegmental area (VTA) and projects to the ventral pallidum [9-12]. The ventral pallidum sends axonal projections to the dorsomedial thalamic nucleus, which then projects to the prefrontal cortex to close the loop $[13,14]$. The dopaminergic input from the VTA modulate the activity of this loop [15]. The Acb is considered to be involved in cognition, emotion and emotional behaviors such as pleasure, fear, aggression, addiction and reward $[16,17]$. The limbic striatal loop is, therefore, one of the major targets of studies on the pharmacological actions of anti-psychotic drugs $[18,19]$.

In the present study, we found the frequent and consistent tau accumulation in the Acb in TPD. Tau positive neurons were also found in the Acb in some AD and ondemented aged subjects but much fewer in the majority of such cases. We speculate that the lesions in the Acb play a role in some psychiatric symptoms such as delusion, which is often conspicuous in TPD.

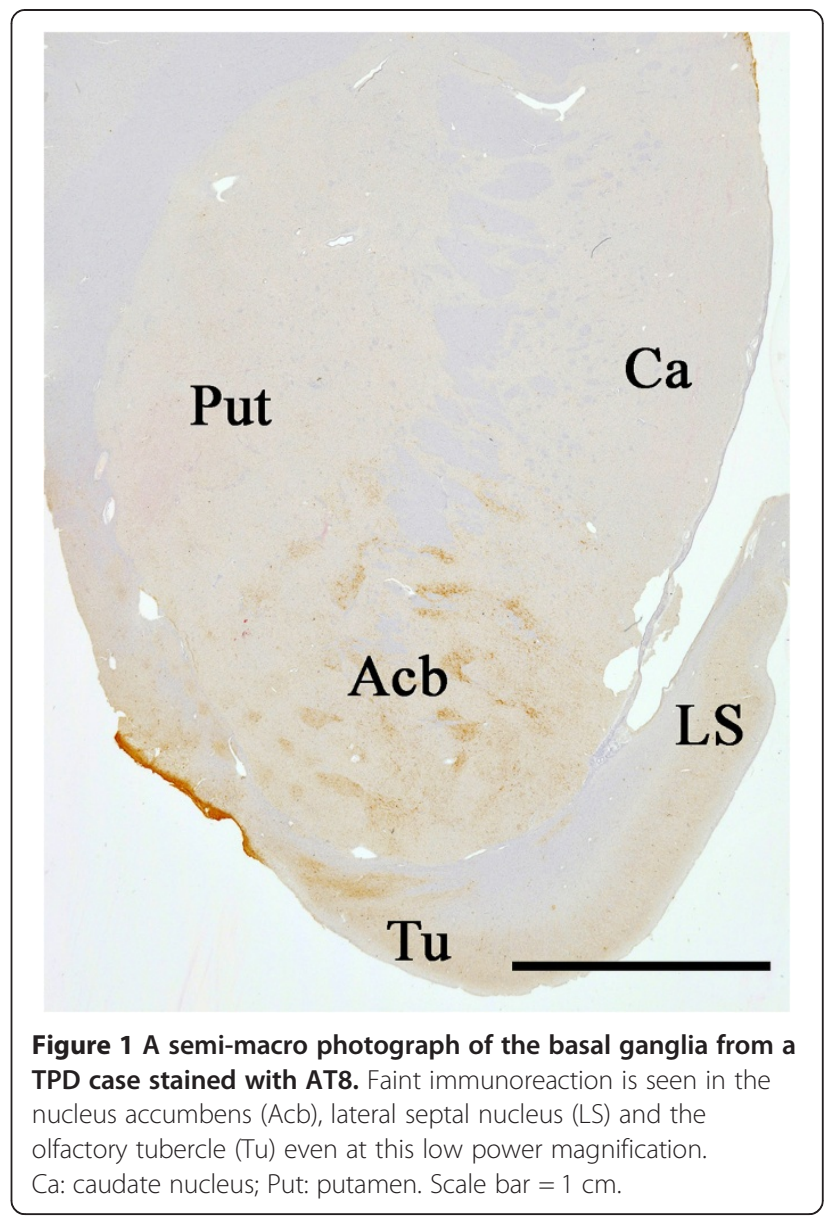

\section{Materials and methods}

We used brain tissues, archived in our laboratory, from 7 patients with TPD, 22 with AD and 11 subjects without dementia or other neurological disease. The demography, Braak and Braak's NFT stages and brain weight in each patient group are summarized in Table 1. Diagnoses were initially made on a clinical basis and were confirmed in every case by neuropathological examination. Clinical and neuropathological diagnoses of TPD followed the descriptions in previous articles [3,7,20]. Diagnoses of $\mathrm{AD}$ were made if the CERAD plaque score was ' $C$ ' [21] and the Braak and Braak's NFT stage was IV or higher [6]. In TPD and AD cases with the NFT stage III or IV, Lewy body pathology was confirmed to be absent or mild/stage 1 [22] in the hippocampus, parahippocampal gyrus and temporal neocortex to exclude the possibility of Dementia with Lewy bodies. In all cases, the patient or, in any case where the patient had died, his/her next of kin gave the written consent for autopsy and postmortem analyses for research purposes. This study was approved by the ethics committee in the Tokyo Metropolitan Institute of Medical Science and was performed in accordance with the ethical standards laid down in the 1964 declaration of Helsinki and its later amendments. 
Table 1 Summary of patient groups used in this study

\begin{tabular}{|c|c|c|c|c|c|}
\hline \multirow[b]{2}{*}{ Braaks' NFT stages } & \multicolumn{2}{|c|}{ TPD } & \multicolumn{2}{|c|}{$A D$} & \multirow{2}{*}{$\begin{array}{c}\text { Non-dementec } \\
\text { I-III }\end{array}$} \\
\hline & III or IV & IV & V & VI & \\
\hline Number of cases & 7 & 10 & 8 & 4 & 11 \\
\hline Gender (male/female) & $1 / 6$ & $6 / 4$ & $4 / 4$ & $2 / 2$ & $7 / 4$ \\
\hline Age at death* & $88.4 \pm 7.2$ & $81.5 \pm 8.5$ & $86 \pm 3.5$ & $81.5 \pm 8.8$ & $81 \pm 7.0$ \\
\hline Disease duration $(y)^{*}$ & $4.7 \pm 2.9$ & $6.1 \pm 6.7$ & $6.2 \pm 3.5$ & $6.6 \pm 6.0$ & n/a \\
\hline Brain weight $(g)^{*}$ & $1,137 \pm 135.3$ & $1,134 \pm 174.8$ & $1,119 \pm 77.2$ & $1,008 \pm 156.5$ & $1,146 \pm 77.1$ \\
\hline
\end{tabular}

$T P D$, Tangle-predominant dementia; $A D$, Alzheimer's disease; NFT, neurofibrillary tangles.

*Data are shown as mean \pm S.D.

For routine neuropathological examinations, formalinfixed, paraffin-embedded brain blocks were cut into $10 \mu \mathrm{m}$ thick sections and stained with hematoxylin and eosin (HE), Klüver-Barrera, modified Gallyas-Braak and methenamine silver staining. Tissue sections of the mediobasal temporal cortex containing the hippocampus, entorhinal cortex and temporal neocortex were stained for tau and amyloid $\beta$ protein $(A \beta)$ by immunohistochemistry. Sections of the rostral striatum with the Acb and the septal nuclei were stained for tau. In TPD cases, additional tau immunohistochemistry was performed for the nucleus basalis of Meynert, amygdala and substantia nigra. The hippocampus, parahippocampal gyrus and adjacent temporal neocortex were also stained for phosphorylated $\alpha$-synuclein and phosphorylated TDP-43 in TPD cases.

For more detailed immunohistochemical analyses, small blocks of brain the tissues were dissected at autopsy and fixed in $4 \%$ paraformaldehyde (PFA) for 2 days. The cryocut sections of $30 \mu \mathrm{m}$ thickness were used for the high sensitive, free-floating immunhistochemical staining [23]. The antibodies used in this study are listed in Additional file 1: Table S1. The primary antibody labeling was visualized with 3,3'-diaminobenzidine as a chromogen, in combination with the Envision Plus ${ }^{\oplus}$ kit (Dako Japan, Tokyo). For enhanced thioflavin-S staining, tissue sections were pretreated with $\mathrm{KMnO}_{4}$ for $20 \mathrm{~min}$ and, subsequently, with sodium borohydride for $4 \mathrm{~min}$ [24]. Sections were then stained with $0.05 \%$ thioflavin-S in $50 \%$ ethanol in the dark for $8 \mathrm{~min}$, followed by differentiation in two changes of $80 \%$ ethanol for $10 \mathrm{sec}$ each time and three washes in large volumes of distilled water. Following incubation in a high salt solution containing $411 \mathrm{mM} \mathrm{NaCl}, 8.1 \mathrm{mM} \mathrm{KCl}$, $30 \mathrm{mM} \mathrm{Na}_{2} \mathrm{HPO}_{4}$ and $5.2 \mathrm{mM} \mathrm{KH}_{2} \mathrm{PO}_{4}, \mathrm{pH} 7.2$ at $4^{\circ} \mathrm{C}$ for $30 \mathrm{~min}$, sections were briefly rinsed with distilled water and observed by fluorescence microscopy.

For immunoelectron microscopy, both post-embedded and pre-embedded procedures were used. For the former, the $4 \%$ PFA-fixed small tissues were embedded in LR White Resin ${ }^{\odot}$ (London Resin, U.K.) without further fixation. The ultra-thin sections were stained with AT8, which was followed by incubation with anti-mouse IgG conjugated with 10 or $20 \mathrm{~nm}$ gold colloidal particles (BBinternational, U.K.). For the pre-embedding procedure, the 4\% PFA-fixed free-floating sections were stained with AT8 in combination with Alexa Fluor 488 FluoroNanogold anti-mouse IgG (Nanoprobes, U.S.A.). Following examination by fluorescence microscopy to localize the positive labeling, the sections were postfixed with $2 \%$ glutaraldehyde and then treated with HQ Silver Enhancement Kit (Nanoprobes, U.S.A.). After the treatment with $1 \%$ osmium tetroxide, which was followed by $2 \%$ uranyl acetate, the sections were embedded in epoxy resin (Querol 812, Nissin EM, Japan). Ultrathin sections were cut and observed by a transmission electron microscope (JEM-1400, JEOL, Japan).

For immunoblot analyses, fresh frozen samples of the Acb and the parahippocampal cortex were obtained from 3 TPD cases (cases 3, 4 and 6) and 3 AD cases. The Braak and Braak's NFT stages of the AD cases were 4, 5 and 6, respectively. Brain tissue was homogenized in 2 vol of TS buffer (50 mM Tris- $\mathrm{HCl}, 150 \mathrm{mM} \mathrm{NaCl}, \mathrm{pH} 7.5$ ), with a mixture of protease inhibitors and centrifuged at 200,000 g for 20 minutes at $4^{\circ} \mathrm{C}$. The supernatant was taken as the soluble fraction and the pellet was used to further extract the sarkosyl-insoluble fraction as described previously [25]. Dephosphorylation of the sarkosyl-insoluble fractions was performed by incubation of the samples with Escherichia coli alkaline phosphatase (type III, Sigma) as described previously [25]. HT7, a pan-tau monoclonal antibody (Additional file 1: Table S1), was used for immunoblotting. Primary antibody labeling on the membranes was visualized with 3,3' -diaminobenzidine as a chromogen, in combination with a Vectastain ABC kit (Vector Lab., USA).

For semiquantitative analyses of immunohistochemically stained tissue sections, the density of AT8 positive tau accumulation was graded to be 0 for absent, 1 for low, 2 for intermediate and 3 for high, based on microscopic observations at $\times 200$ magnification. The Acb, septal nuclei, caudate nucleus, hippocampal CA1, entorhinal cortex and temporal neocortex were assessed in TPD, AD and nondemented aged subjects. The Mann-Whitney $U$ test was used for statistical analyses using Graph Pad Prism 4 software (Graph Pad Software, U.S.A.). 


\section{Results}

\section{TPD cases used in the present study}

The demographic, pathologic, and clinical information of the TPD cases used in the present study is summarized in Tables 1 and 2 . In general, both the clinical and neuropathological features are similar to those described in previous reports [1-3,7,26,27]. The average age at death is higher than that in AD. Moderate dementia was noted in 5 of the 7 cases but the other two were diagnosed as having mild cognitive impairment. Delusion was evident in 6 cases. Brain atrophy was mild, if present, and senile plaques were either absent or rare. Lacunar infarcts were seen in the globus pallidus in 2 cases. In all cases, heavy tau accumulation was seen in the limbic regions in the forms of NFT, diffuse cytoplasmic accumulations and neuropil threads. Tau accumulation was heavier in the subiculum and the CA1 region than in the entorhinal and transentorhinal cortices. Tau was also deposited in the amygdala, the septal nuclei and the basal nucleus of Meynert, and, less frequently, in the caudate nucleus and substantia nigra. A small amount of tau was found in the temporal neocortex but only in 3 cases. Such limbic-predominant distribution of tau pathology is consistent with previous reports $[1,2,26,28]$. A small number of argyrophilic grains were present in 2 cases.

\section{Tau accumulation in the Acb in TPD}

In addition to the previously reported tau distribution, we found a considerable number of tau positive neurons in the Acb in all TPD cases used in this study (Figures 1 and 2). Similarly to the hippocampus, numerous neuropil threads were associated with tau positive neurons (Figure 2A). The tau positive neurons and neuropil threads were labeled with all the anti-tau antibodies used in the present study (Figures 2A-D). They included conformational change-specific, phosphorylation-specific and phosphorylation-independent antibodies (Additional file 1: Table S1). The staining pattern varied, which partly depends on the affinity of the antibody and the localization of the antigen epitope recognized by each antibody. Preservation of the epitope in tissue sections is affected by aggregation, degradation and post-mortem processing such as fixation. The majority of tau positive neurons in the Acb showed pretangle-like, diffuse or granular accumulation of tau in the cytoplasm (Figures 2B). Flame-like NFT, the common form in the hippocampus in TPD, were also present but not frequent (Figure 2B, arrow). The vast majority of tau positive neurons were medium sized but, occasionally, large neurons were also stained positively for tau (Figure 2D, arrow). Tau positive neurons and threads were not distributed evenly in the Acb. Rather, areas with sparseand dense-tau pathology were intermingled (Figure 2E).

Table 2 Demography and basic clinical and neuropathological features of TPD cases

\begin{tabular}{|c|c|c|c|c|c|c|c|}
\hline & Case 1 & Case 2 & Case 3 & Case 4 & Case 5 & Case 6 & Case 7 \\
\hline Age at death & 89 & 102 & 90 & 85 & 89 & 78 & 86 \\
\hline Sex & $\mathrm{F}$ & $\mathrm{F}$ & $\mathrm{F}$ & $\mathrm{F}$ & $F$ & $M$ & $\mathrm{~F}$ \\
\hline Dementia & + & + & + & + & $\mathrm{MCl}$ & $\mathrm{MCl}$ & + \\
\hline \multicolumn{8}{|l|}{ Psychiatric symptoms } \\
\hline Delusion & + & + & + & + & + & + & - \\
\hline Anxiety & + & - & - & - & - & - & - \\
\hline Depression & - & - & - & + & - & - & - \\
\hline Brain weight (g) & 940 & 970 & 1170 & 1300 & 1230 & 1220 & 1130 \\
\hline Atrophy & $\operatorname{mi}(\mathrm{Fr})$ & $\mathrm{mi}(\mathrm{Fr} / \mathrm{T})$ & - & - & - & - & - \\
\hline Plaque stage (1) & 0 & 0 & $A^{*}$ & 0 & 0 & 0 & 0 \\
\hline NFT stage (1) & III & III & III & III & IV & III & III \\
\hline Argyrophilic grain stage (2) & 0 & 0 & 0 & 0 & $\|$ & $\|$ & 0 \\
\hline Hippocampal sclerosis & - & - & + & - & - & - & - \\
\hline Vascular lesions & + & - & - & + & - & - & - \\
\hline a-synuclein (hip/T**) & $+\S$ & - & - & - & - & - & - \\
\hline TDP-43 (hip/T**) & - & - & - & - & - & - & - \\
\hline Acb tau score & 3 & 2 & 2 & 3 & 3 & 2 & 3 \\
\hline
\end{tabular}

F, Female; $M$, Male; $M C l$, Mild cognitive impairment; $m i$, Mild; $F r$, Frontal; $T$, Temporal; $A c b$, The nucleus accumbens. ${ }^{*} \mathrm{~A}$ small number of diffuse $\mathrm{A} \beta$ deposits were seen in the temporal cortex. The Acb tau score was determined according to the method described in the text. (1) The senile plaque and NFT staging were based on the description by Braak and Braak [6]. (2) The argyrophilic grain staging was based on the description by Saito et al. [29]. **Immunohistochemistry for a-synuclein and TDP-43 was performed in tissue sections of the hippocampus, parahippocampal gyrus and adjacent temporal neocortex. $\S$ a-Synuclein pathology in this case was mild, corresponding to stage 1 by the $3^{\text {rd }}$ report of the DLB consortium [22]. 


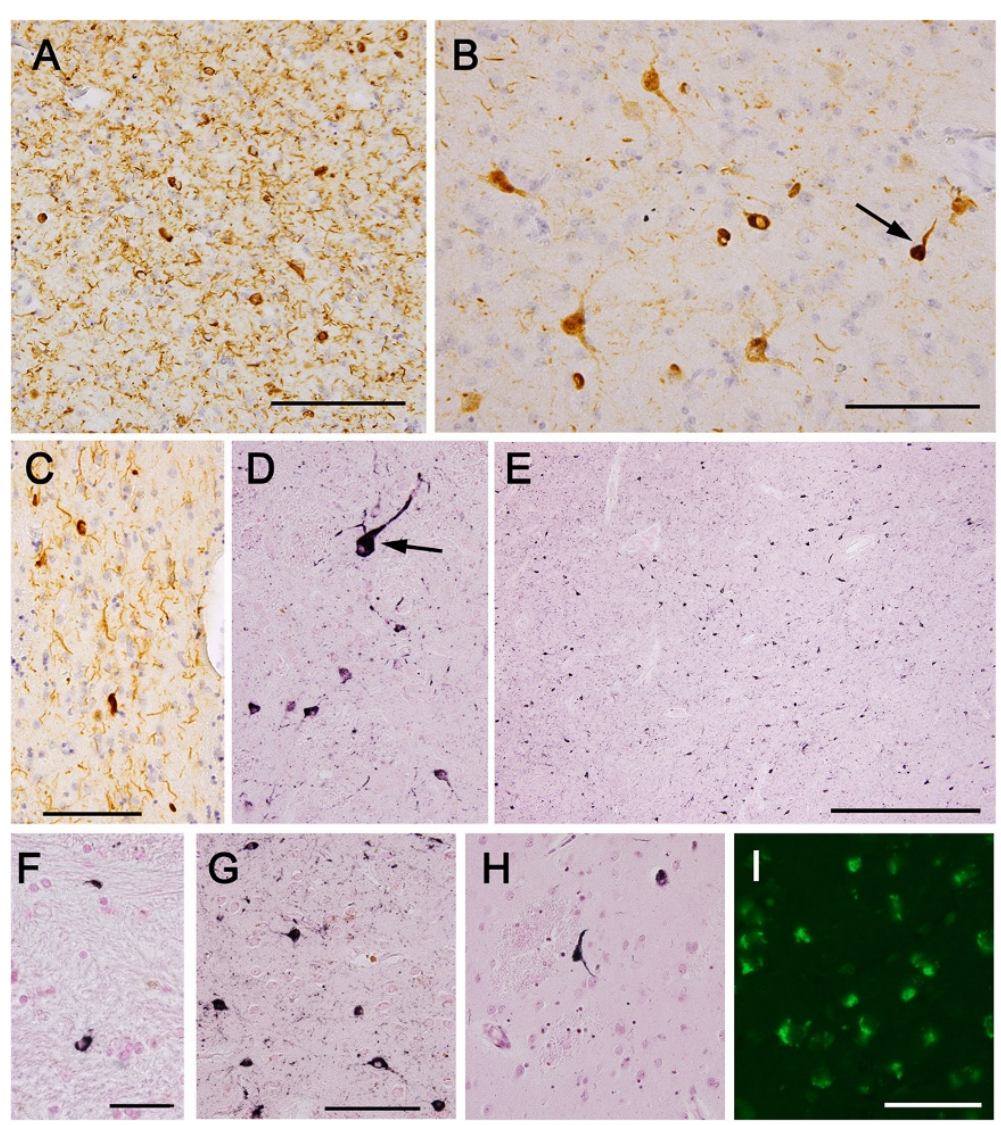

Figure 2 Tau accumulation in the Acb in TPD. A through $\mathbf{G}$ are immunohistochemistry with phosphorylation or conformational change specific tau antibodies. A, B, C and I: 4\% paraformaldehyde-fixed, frozen-cut, $30 \mu \mathrm{m}$ thick Sections. D through $\mathbf{H}$ are formalin-fixed, $10 \mu \mathrm{m}$ thick, paraffin Sections. A: immunohistochemistry with AP422. Tau positive neurons are associated with many neuropil threads. Scale bars $=200 \mu \mathrm{m}$. B: immunohistochemistry with PHF-1. The majority of tau positive neurons show pretangle-like, diffuse or granular cytoplasmic labeling. Among them, apparent NFT are also seen but less frequently (arrow). Scale bar $=100 \mu \mathrm{m}$. C: immunohistochemistry with MC1, a conformational change specific antibody. Scale bar $=100 \mu \mathrm{m}$. $\mathbf{D}$ through $\mathbf{G}$ are immunohistochemistry with AT8. D: the vast majority of tau positive neurons are of medium-size but, occasionally, large neurons are also stained positively for tau (arrow). At the same magnification as $\mathbf{C}$. E: tau positive neurons are not evenly distributed in the Acb. Scale bar $=500 \mu \mathrm{m}$. F: a glial coiled body. Scale bar $=25 \mu \mathrm{m}$. $\mathbf{G}$ and $\mathbf{H}$ : the nearby sections from the same case with AT8 immunohistochemistry (G) and Gallyas-Braak staining (H). I: thioflavin S staining reveals granular cytoplasmic labeling of neurons. Scale bar $=100 \mu \mathrm{m}$.

Occasional glial coiled bodies were seen in the majoriy, if not all, of the cases (Figure 2F). Occurrence of glial coiled bodies in other brain regions in TPD has been reported previously [3]. Gallyas-Braak staining labeled only a small number of NFT in the Acb, while tau immunohistochemistry of nearby sections from the same patient revealed many positive cells (Figure $2 \mathrm{G}$ and $2 \mathrm{H}$ ). Enhanced thioflavin-S staining labeled many neurons (Figure 2I).

The density of tau positive neurons and neuropil threads varied somewhat among the TPD cases. In TPD, no clear association was seen between the degree of Acb tau pathology and the Braak and Braak's NFT stage or the presence or absence of $A \beta$ deposits, argyrophilic grains [29] and vascular lesions (Table 2). Despite the consistent tau accumulation in the Acb in TPD, we were not able to find severe neuronal loss or gliosis by HE staining.
Immunoelectron microscopy of the Acb in TPD with a tau antibody, AT8, revealed positive labeling of granular structures in the neurons (Figure 3A). Small and sparse bundles of short filamentous structures were occasionally seen to be stained positively for AT8 in the neuronal cytoplasm and neuropil (Figure 3B). Some of them showed morphology consistent with paired helical filaments (PHF). Thus, the ultrastructure of tau accumulation in the Acb was different from that in the hippocampal CA1 region, where dense and long bundles of PHF were frequent and intensely labeled for AT8 (Figure 3C).

\section{Tau pathology in the Acb in AD and non-demented aged subjects}

We then investigated the Acb in $\mathrm{AD}$ and non-demented, aged subjects. Tau positive neurons were found in some, 


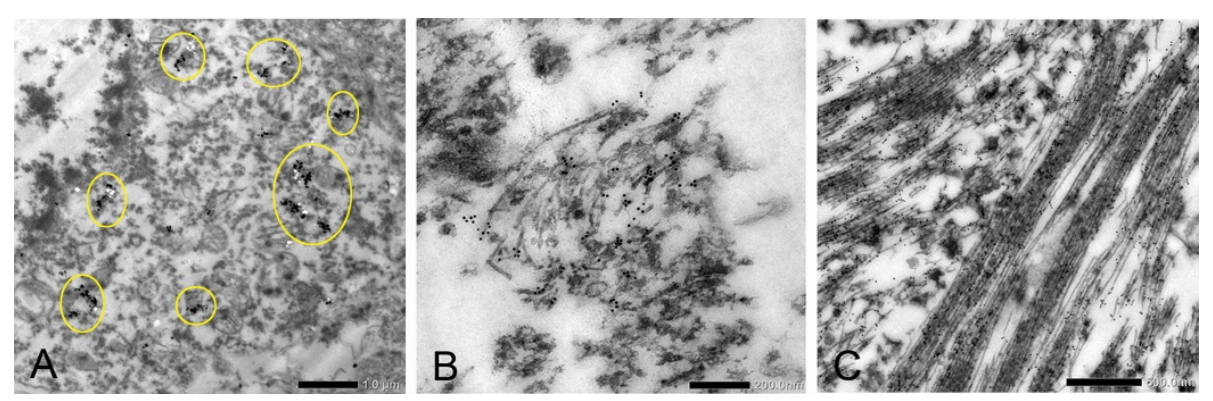

Figure 3 Immunoelectron microscopy of TPD brain with AT8 and immunogold labeling. A: in the Acb, the immunogold labeling in neurons was mostly localized to the granular structures (indicated by circles). Paired-helical filaments (PHF) were rare. Scale bar $=1.0 \mu \mathrm{m}$. B: sparse bundles of PHF were scattered in the neuronal cytoplasm. Scale bar $=200 \mathrm{~nm}$. C: in the hippocampal CA1 region, prominent bundles of AT8 positive PHF were seen. Scale bar $=500 \mathrm{~nm}$.

but not all, AD patients and non-demented, aged subjects (Additional file 2: Figure S1A). In these groups, however, only a limited number of cases showed tau pathology which was similarly abundant to that in TPD (Figure 4). In $\mathrm{AD}$ patients with heavy tau accumulation in the Acb, the caudate nucleus was also affected, a feature which distinguished $\mathrm{AD}$ from TPD. In TPD, the caudate tau lesions were either absent or, if present, very mild in all cases. In addition, senile plaques with tau positive dystrophic neurites were scattered in the Acb of such AD cases (Additional file 2: Figure $\mathrm{S} 1 \mathrm{~B}$ ). In $\mathrm{AD}$ cases with mild tau pathology in the Acb, large neurons preferentially contained tau, a finding which was similar to the caudate nucleus in $\mathrm{AD}$. In $\mathrm{AD}$ cases with heavy tau pathology in the Acb, such large neuron predominance became unclear and many tau

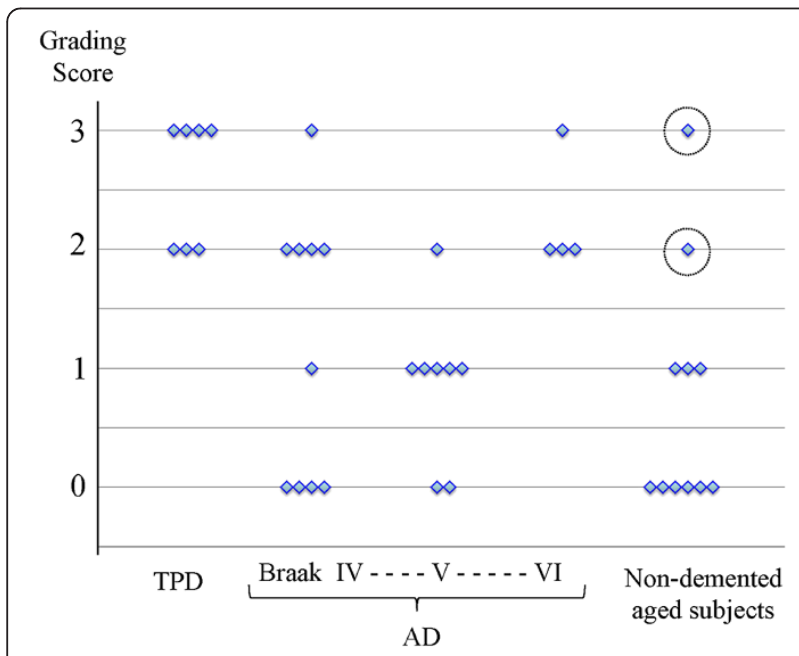

Figure $4 \mathrm{~A}$ graph of the density of neuronal tau accumulation in the Acb. The density of tau accumulation was graded as being 0 for absent, 1 for low, 2 for intermediate and 3 for high. Statistically significant differences were seen between TPD cases and non-demented, aged subjects ( $P=0.0031)$ as well as AD cases in NFT stage IV $(P=0.0192)$ and $V(P=0.0022)$. The points encircled by a broken line in the non-demented, aged group indicate that the cases were over age 90 . positive, medium-sized neurons were seen. In both $\mathrm{AD}$ and non-demented, aged subjects, neuropil threads were also present in those with tau positive neurons in the Acb (Additional file 2: Figure S1C). The form of tau accumulation in $\mathrm{AD}$ patients and non-demented, aged subjects was similar to that in TPD, being predominantly pre-tangle like, diffuse accumulation in the cytoplasm.

The density of neuronal tau accumulation was graded to be 0 (absent) through 3(high) in AT8 immunostained tissue sections. Figure 4 illustrates the results in the Acb. Tau density in the Acb in the $\mathrm{AD}$ group was highly variable, except that the cases in Braak and Braak's NFT stage VI were either grade 2 or 3 . Statistically significant differences were seen between the TPD cases and the non-demented, aged subjects $(P=0.0031)$ as well as the AD cases with NFT stage IV $(P=0.0192)$ and those with NFT stage V $(P=$ $0.0022)$. Two non-demented, aged subjects with tau accumulation in the Acb were both over age 90. These 2 cases, similarly to TPD, lacked tau accumulation in the caudate nucleus and showed more NFT in the subiculum than in the entorhinal cortex. The results of semiquantitative analyses confirmed our observation that tau accumulation in the Acb was a remarkable finding in TPD. We performed similar analyses for a number of brain regions. The results are summarized in Table 3 as the averages of the graded scores for tau accumulation in each group. The concentration of tau pathology in the limbic structures, including the Acb and septal nuclei, in TPD contrasted with the broad distribution over the neocortex in $\mathrm{AD}$.

\section{Immunoblot analyses}

The results of immunoblot analyses of samples from TPD and $\mathrm{AD}$ patients are shown in Figure 5. The tau band patterns in the sarkosyl insoluble fraction appeared to be essentially the same between TPD and AD, while the amount of insoluble tau was far smaller in the Acb than in the parahippocampal cortex in $\mathrm{AD}$. It has to be noted that, because of the very high concentrations of insoluble tau in the parahippocampal cortex samples, the amounts of samples 
Table 3 Summary of the semiquantitative grading of tau accumulation

\begin{tabular}{lcccccccc}
\hline & Braak stage & No. of cases & Acb & Caudate nucleus & Septal nucleus & CA1 & Ent & Temp \\
\hline TPD & III-IV & 7 & 2.6 & 0.7 & 2.2 & 0 & 3 & 2.57 \\
Non-demented & I-II & 3 & 0 & 0 & 0.4 & 0.67 & 1 & 0 \\
& III & 8 & 1.13 & 0.25 & 0.63 & 1.38 & 2 & 1 \\
AD & IV & 10 & 1.2 & 0.6 & 1.4 & 2.67 & 3 & 1.4 \\
& V & 8 & 0.9 & 0.9 & 1.3 & 2.89 & 2.9 & 2.5 \\
& VI & 4 & 1.3 & 1.3 & 1.7 & 3 & 3 \\
\hline
\end{tabular}

The numbers indicate averages of the scores in each group. The degree of tau pathology was qualitatively scored as 0: absent 1: low 2: intermediate 3: high. $T P D$, Tangle predominant dementia; AD, Alzheimer's disease; NFT, Neurofibrillary tangles; Acb, nucl. Accumbens; CA1, Hippocampal CA1 region; Ent, Entorhinal cortex; Tmep, Temporal neocortex.

applied to the gels had to be reduced in AD cases. This resulted in the relatively weak signals for the Acb samples in AD cases. The dephosphorylated samples of the Acb and parahippocampal cortex showed the $3 R+4 R$ isoform pattern in both TPD and AD.

\section{The Acb tau pathology and the presence/absence of clinical history of delusion}

Finally, we examined if the degree of the Acb tau pathology was different between the subjects groups with and without the history of delusion in the clinical records (Additional file 3: Figure S2). In the group of subjects with Braaks' NFT stages III and IV, which included NFT stage III non-demented aged subjects, all TPD cases and NFT stage IV AD cases, the Acb tau score was higher in those with clinical history of delusion than those without it $(p=0.033)$. Similarly, the less conspicuous but still significant difference was seen in the group of all NFT stage $\operatorname{AD}$ cases $(p=0.049)$.

\section{Discussions}

There is significant overlap in the distribution of NFT between TPD and AD. However, an early genetic study of TPD cases indicated a paucity of the apolipoprotein E $\varepsilon 4$ allele, which currently is the most powerful risk factor for $\mathrm{AD}$ [30]. More recently, a report has been made on the significant associations of TPD with the MAPT H1 haplotype as well as with some polymorphisms within the region of MAPT encoding the 3' UTR [31]. Thus, together with the striking paucity of $A \beta$ deposition, it seems that TPD is a unique neuropathological entity that has to be studied separately from AD.

The clinical and neuropathological features of the TPD patients we used in the present study generally agreed with those described in preceding articles [3,32]. In
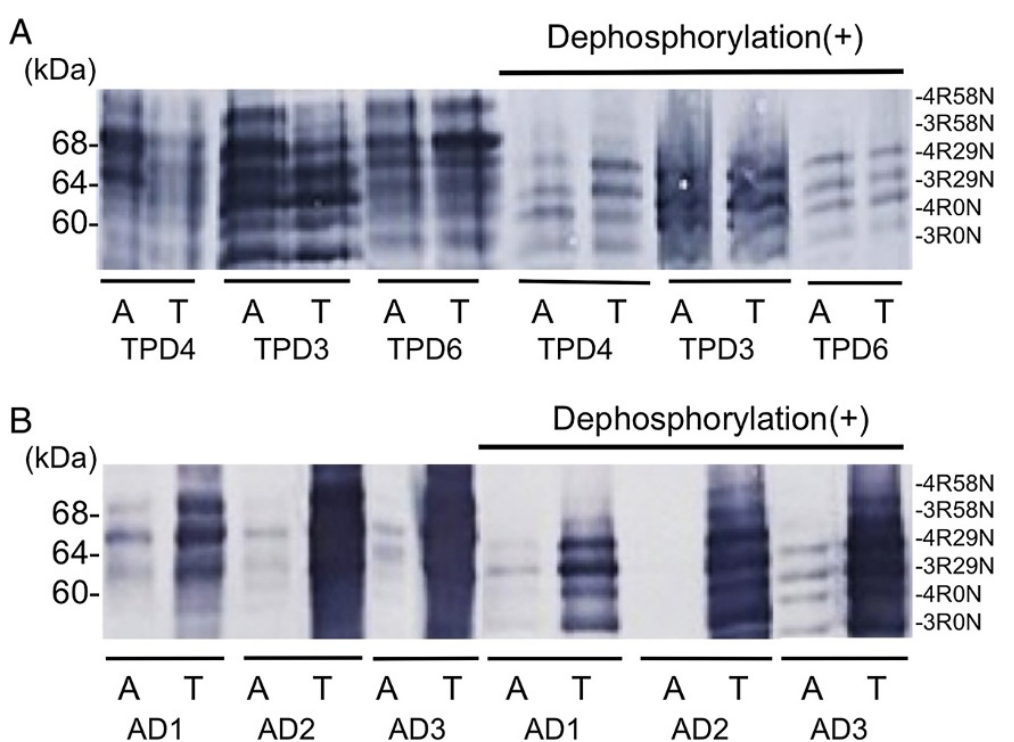

Figure $\mathbf{5}$ Immunoblot analyses of the sarkosyl insoluble tau. The sarkosyl insoluble fractions of the Acb (A) and the parahippocampal cortex (T) from TPD (A) and AD (B) were analyzed by immunoblot. A pan-tau antibody, HT7, was used. The Acb samples show the 3R + 4R isoform pattern similar to that in the parahippocampal cortices in both TPD and AD. 
addition to the already well-known distribution of tau pathology, we found a considerable number of tau positive neurons and neuropil threads in the Acb. The Acb consists of the predominant medium-sized neurons and the occasional large neurons. Both cell types were affected by tau pathology in TPD. This contrasted AD with mild Acb tau pathology, in which large neurons were affected preferentially. Such a result is consistent with the previous reports which described that large neurons are more vulnerable in $\mathrm{AD}$ [33] and prone to tau accumulation [34]. In AD cases with the heavy Acb tau pathology, many medium-sized neurons were also tau positive. In the absence of TPD cases with the mild Acb tau pathology, it remains to be determined whether the difference is attributable to the distinct pathomechanisms between $\mathrm{AD}$ and TPD or to the variable vulnerability of different neuronal cell types.

The Acb may not be a region which is routinely sampled in a number of laboratories. Further, tau pathology in the Acb is not well stained by the Gallyas-Braak method. These facts together might explain the absence of previous reports on the occurrence of tau lesions in the Acb. The mechanism by which the Gallyas-Braak staining labels NFT remains to be determined. In TPD, insoluble tau consists of a mixture of the $3 \mathrm{R}$ and $4 \mathrm{R}$ isoforms in both the Acb and hippocampus, but NFT are intensely labeled by Gallyas-Braak staining in the latter. It may be noteworthy that, in the Acb, tau pathology occurs in a form of diffuse or granular cytoplasmic accumulations in the majority of tau positive neurons and that, ultrastructurally, PHF were rare. These contrasted with the hippocampal lesions where dense bundles of PHF were frequently seen. Obviously, factors that affect the reactivity of abnormal tau deposits to Gallyas-Braak staining need further clarification.

Tau pathology is considered to propagate in the brain from an affected region to another along the fiber connections, by spreading through the neuropil, or by both. A proposed mechanism for such propagation is a prion-like, seed-dependent conformational change and subsequent aggregation of the molecule, with a breakdown of the aggregate that generates the next seeds. In such a manner, the tau isoform pattern in the initial aggregates may be maintained in the later aggregate formations [35]. In the present study, we found that the Acb lesion in TPD was $3 R+4 R$ tauopathy, a result which suggests the common origin of the tau pathology in the Acb with that in the hippocampus. The prevalence of diffuse cytoplasmic accumulations suggests that tau pathology in the Acb occurs later in the disease progression. In the hippocampus in TPD, many ghost tangles are seen, suggesting that the hippocampal lesions precede the Acb lesions.

Occurrence of NFT in the Acb in AD was reported previously $[36,37]$. In the present study, however, we have found that tau accumulation in TPD is more frequent and consistent than AD. The Acb receives direct and massive projections from the hippocampal CA1 and subiculum $[14,38,39]$. It has been repeatedly reported in TPD that the density of NFT is higher in the hippocampal CA1 and subiculum than in the entorhinal cortex $[1,2,7,28]$. Thus, the heavy tau pathology in the subiculum and CA1, through neural circuit-mediated propagation to the Acb, may result in the more pronounced tau accumulation in the Acb in TPD than in AD. Such an idea may be consistent with our finding that the difference in Acb tau pathology was statistically significant between TPD and AD in NFT stages IV and V but not in AD at stage VI. In AD with Acb lesions, tau accumulation was also found more frequently in the caudate nucleus than was the case in TPD. The caudate nucleus receives massive innervations from the cerebral cortex where, unlike TPD, tau pathology is severe in $\mathrm{AD}$. On the other hand, the septal nuclei, like the Acb, receive direct projections from the subiculum and CA1 $[14,38]$. Again, we found, in the present study, heavier tau accumulation in these areas in TPD than in $\mathrm{AD}$ at NFT stages IV and V.

TPD is primarily an amnestic disease with relatively mild non-amnestic symptoms of dementia. In 6 of 7 TPD cases used in this study, however, delusion was a consistent clinical feature. This may be partly attributable to the fact that our brain tissue archive is principally based on the psychiatric hospital autopsies. However, occurrence of psychiatric symptoms has also been described in a number of previous reports on TPD. As an example, Jellinger et al. reported depression in $17.5 \%$ and paranoid ideas in $15 \%$ of TPD cases [3]. The Acb is part of the mesolimbic system in which the Acb receives dopaminergic input from the VTA. Recent evidence suggests that, in schizophrenia, functional abnormality in the Acb causes excessive release of dopamine from the VTA, which then results in the psychiatric symptoms [19,40-43]. While neuronal loss was not apparent in the Acb in TPD, it may be noteworthy that association of intraneuronal tau aggregation with clinical symptoms has been suggested in early stage AD lesions [44]. In AD, cases with more neocortical NFT were reported to be associated with more psychosis [45]. Thus, tau accumulation in the Acb could be related to the frequent delusion in TPD. Delusion and other psychotic symptoms may occur by multiple mechanisms in dementia patients. We have to note that 2 of the 7 TPD cases had argyrophilic grain pathology and that psychotic symptoms are known to be common in the patients with argyrophilic grain disease [46]. Whether the Acb tau accumulation is related to the psychiatric symptoms in TPD may be an issue for further investigation.

In the present study, we found that tau pathology occurred unevenly in the Acb in TPD (Figures 1 and 2E). The striatum is not uniform and has distinct neurochemical 
compositions and connections that are referred to as matrix and striosomes. The similar but more complex compartmentation was reported in the human Acb [47]. We have performed additional immunohistochemistry for tyrosine hydroxylase $(\mathrm{TH})$ and tau in serially-cut, free-floating sections in two TPD cases, in which the remnants of Acb blocks were available after the initial sectioning for the main body of this study. Comparison of the adjacent sections stained for $\mathrm{TH}$ and tau indicates that tau pathology preferentially occurs in areas where the fine, mesh-like $\mathrm{TH}$ staining is relatively light (Additional file 4: Figure S3). Such a result suggests the relationship between the uneven distribution of tau pathology and neurochemical heterogeneity in the Acb. However, because of the limited number of currently available samples and of the more complex neurochemical architecture in the Acb than the simple matrixstriosome structure in the caudate nucleus [47], future, extensive studies should be needed for further exploration.

\section{Conclusions}

We have found frequent tau accumulation in the Acb in patients with TPD. Both the medium-sized and large neurons are affected. While similar tau accumulation was seen in a small number of all AD patients, it was far more frequent and consistent in TPD than AD. The tau isoforms abnormally accumulated in the Acb were $3 R$ and $4 R$, which suggests a common origin with the hippocampal tau pathology. The Acb receives direct and massive projections from the hippocampal CA1 and subiculum where tau pathology is extremely severe in TPD. Such a result may support the idea that abnormal tau aggregation propagates via neural circuits. Tau accumulation in TPD should be a subject of further investigations to approach the long-lasting issue of the simultaneous deposition of $\mathrm{A} \beta$ and tau in $\mathrm{AD}$. In addition, the relationship between the tau pathology in the Acb and such psychiatric symptoms as delusion in TPD needs further exploration.

\section{Additional files}

Additional file 1: Table S1. The primary antibodies used in this study. Additional file 2: Figure S1. Tau accumulation in the Acb in AD and non-demented, aged subjects. Immunohistochemistry with AT8. A: absence of tau positive neurons in an AD case in Braak and Braak's NFT stage IV. Scale bar $=100 \mu \mathrm{m}$ in A-C. B: a diffuse cytoplasmic staining, neuropil threads and duystrophic neurite in a senile plaque in an AD case in NFT stage VI. C: a tau positive neuron and neuropil threads in a non-demented, aged subject.

Additional file 3: Figure S2. A graph of the density of neuronal tau accumulation in the Acb. The left plots: the group of Braaks' NFT stages III and IV, which includes non-demented aged subjects, TPD cases and AD cases with Braaks' NFT stage IV. Cases with delusion in the clinical history show higher tau score than those without delusion in the Acb. The right plots: the group of AD cases with Braaks' NFT stages IV through VI. Again, cases with delusion show higher tau score than those without delusion.
Additional file 4: Figure S3. The serial section immunohistochemistry for tau and tyrosine hydroxylase (TH). Forty micrometer thick, free floating sections were cut serially from two tangle predominant dementia (TPD) cases, in which the remnants of Acb blocks were available after the initial sectioning for the main body of the study. A set of every other section was stained for $\mathrm{TH}$ and the other set for tau with AT8. A and C: AT8 staining in a TPD case 1. B: TH staining of the section between $A$ and $C$. In B, two types of areas are distinguished based on the modest difference in the density of fine, mesh-like TH staining. There is a propensity that tau pathology preferentially occurs in areas where the fine, mesh-like TH staining is relatively light $(A, C)$. Scale bar $=2 \mathrm{~mm}$ in $A$ ( $A, B$ and $C$ are at the same magnification). D: higher power photomicrographs of the boxed areas in B and C. The left half is the staining with AT8 and the right half staining for $\mathrm{TH}$. Scale bar $=400$ micro-m (D).

\section{Abbreviations}

(TPD): Tangle-predominant dementia; (NFT): Neurofibrillary tangles; (AD): Alzheimer's disease; (3R): 3-repeat; (4R): 4-repeat; (Acb): Nucleus accumbens; (VTA): Ventral tegmental area; (HE): Hematoxylin and eosin; (PFA): Paraformaldehyde; (PHF): Paired helical filaments; (AB): Amyloid $\beta$ protein; (TH): tyrosine hydroxylase.

\section{Competing interests}

The authors declare that they have no competing interests.

\section{Authors' contributions}

IK carried out the microscopic observation, immunoblot and statistical analyses. IK also drafted the initial manuscript. MHa conducted the sample preparation and immunoblot and carried them out with IK. TA participated in the design and coordination of the study. $\mathrm{KI}$ carried out the microscopic observation with $\mathrm{IK} . \mathrm{KO}, \mathrm{KN}$ and NA organized the brain archives including clinical information, selected appropriate cases, and performed neuropathological analyses of all cases used in this study. OK participated in the design of the study and performed statistical analyses with IK. SH conceived of the study and participated in the initial design. $\mathrm{MHO}$ contributed to the reagents, materials and analysis tools, and conducted free-floating immunohistochemistry. YH participated in the design of the study and helped to draft the manuscript. HA supervised the design and coordination of the study and worked up the manuscript. All authors read and approved the final manuscript.

\section{Authors' information}

IK is a graduate student of Department of Psychiatry, Graduate school of Medicine, Yokohama City University School of Medicine. MHa is the senior director of Department of Neuropathology and Cell Biology, Tokyo Metropolitan Institute of Medical Science (TMIMS). TA, KI, NA and SH contributed to this study as visiting scientists of Dementia project, TMIMS. TA is also an associated professor of Department of Psychiatry, Graduate School of Comprehensive Human Sciences, University of Tsukuba. KO and KN are psychiatrists and neuropathologists in Tokyo Metropolitan Matsuzawa Hospital (TMMH), visiting scientists of Dementia project, TMIMS and in charge of the brain archive of TMMH/TMIMS. OK is an associate professor of Department of Psychiatry, Graduate school of Medicine, Yokohama City University School of Medicine. MHo is a chief researcher of Dementia project, TMIMS. YH is a professor of Department of Psychiatry, Graduate school of Medicine, Yokohama City University School of Medicine. HA is the senior director of Dementia project, TMIMS.

\section{Acknowledgements}

We are grateful to the patients and their families who made this research possible. We thank Dr. Peter Davies (Albert Einstein College of Medicine) for providing us with his excellent anti-tau antibodies, PHF-1 and MC-1. We also thank Ms. Yoko Shimomura and Mr. Kentaro Endo (Tokyo Metropolitan Institute of Medical Science) for their technical assistance with immuno-electron microscopy, and Ms. Yoko Shimomura, Ms. Hiromi Kondo and Ms. Chie Haga (Tokyo Metropolitan Institute of Medical Science) for their assistance in tissue preparation and staining. Helpful advice by Dr. Takashi Nonaka (Tokyo Metropolitan Institute of Medical Science), as well as Dr. Takashi Togo and Dr. Masatake Uchikado (Department of Psychiatry, Yokohama City University School of Medicine), is very much appreciated. None of the 
authors has any conflict of interest. This research was supported by Grantsin-Aid from the Ministry of Health, Labor and Welfare (12946221, MH, MH and HA) and Ministry of Education, Culture, Science (24500429, HA), Japan.

\section{Author details}

'Dementia Research Project, Tokyo Metropolitan Institute of Medical Science, 2-1-6 Kamikitazawa, Setagaya-ku, Tokyo 156-8506, Japan. ${ }^{2}$ Department of Psychiatry, Graduate school of Medicine, Yokohama City University School of Medicine, Yokohama, Japan. ${ }^{3}$ Department of Neuropathology and Cell Biology, Tokyo Metropolitan Institute of Medical Science, Tokyo, Japan. ${ }^{4}$ Department of Psychiatry, Graduate School of Comprehensive Human Sciences, University of Tsukuba, Tsukuba, Japan. ${ }^{5}$ Department of inflammation of Pathology, Faculty of Medicine, Kagawa University, Takamatsu, Japan. ${ }^{6}$ Tokyo Metropolitan Matsuzawa Hospital, Tokyo, Japan.

Received: 16 February 2014 Accepted: 1 April 2014

Published: 8 April 2014

\section{References}

1. Ikeda K, Akiyama H, Arai T, Oda T, Kato M, Iseki E, Kosaka K, Wakabayashi K, Takahashi H (1999) Clinical aspects of 'senile dementia of the tangle type'- a subset of dementia in the senium separable from late onset. Alzheimer's disease. Dement Geriatr Cogn Disord 10:6-11

2. Noda K, Sasaki K, Fujimi K, Wakisaka Y, Tanizaki Y, Wakug- awa Y, Kiyohara Y, lida M, Aizawa H, Iwaki T (2006) Quantitative analysis of neurofibrillary pathology in a general population to reappraise neuropathological criteria for senile dementia of the neurofibrillary tangle type (tangle-only dementia) the Hisayama Study. Neuropathology 26:508-518

3. Jellinger KA, Attems J (2007) Neurofibrillary tangle-predominant dementia: comparison with classical Alzheimer disease. Acta Neuropathol 113:107-117

4. McKhann GM, Albert MS, Grossman M, Miller B, Dickson D, Trojanowski JQ, Work Group on Frontotemporal Dementia and Pick's Disease (2001) Clinical and pathological diagnosis of frontotemporal dementia: report of the work group on frontotemporal dementia and Pick's disease. Arch Neurol 58:1803-1809

5. Cairns NJ, Bigio EH, Mackenzie IR, Neumann M, Lee VM, Hatanpaa KJ, White CL 3rd, Schneider JA, Grinberg LT, Halliday G, Duyckaerts C, Lowe JS, Holm IE, Tolnay M, Okamoto K, Yokoo H, Murayama S, Woulfe J, Munoz DG, Dickson DW, Ince PG, Trojanowski JQ, Mann DM (2007) Neuropathologic diagnostic and nosologic criteria for frontotemporal lobar degeneration: consensus of the Consortium for Frontotemporal Lobar Degeneration. Acta Neuropathol 114:5-22

6. Braak H, Braak E (1991) Neuropathological staging of Alzheimer-related changes. Acta Neuropathol 82:239-259

7. Yamada M (2003) Senile dementia of the neurofibrillary tangle type (tangle-only dementia): neuropathological criteria and clinical guidelines for diagnosis. Neuropathology 23:311-317

8. Kovacs GG, Molnár K, László L, Ströbel T, Botond G, Hönigschnabl S, ReinerConcin A, Palkovits M, Fischer P, Budka H (2011) A peculiar constellation of tau pathology defines a subset of dementia in the elderly. Acta Neuropathol 122:205-222

9. Groenewegen HJ, Vermeulen-Van der Zee E, te Kortschot A, Witter MP (1987) Organization of the projections from the subiculum to the ventral striatum in the rat. A study using anterograde transport of Phaseolus vulgaris leucoagglutinin. Neuroscience 23:103-210

10. Kita H, Kitai ST (1990) Amygdaloid projections to the frontal cortex and the striatum in the rat. J Comp Neurol 298:40-49

11. Berendse HW, Galis-de Graaf Y, Groenewegen HJ (1992) Topographical organization and relationship with ventral striatal compartments of prefrontal corticostriatal projections in the rat. J Comp Neurol 316:314-347

12. French SJ, Totterdell S (2002) Hippocampal and prefrontal cortical inputs monosynaptically converge with individual projection neurons of the nucleus accumbens. J Comp Neurol 446:151-165

13. Heimer L, Zahm DS, Churchill L, Kalivas PW, Wohltmann C (1991) Specificity in the projection patterns of accumbal core and shell in the rat. Neuroscience 41:89-125

14. Groenewegen HJ, Berendse HW, Meredith GE, Haber SN, Voorn P, Wolters JG, Lohman AHM (1991) Functional anatomy of the ventral limbic systeminnervated striatum. In: Willner P, Scheel-Kruger J (eds) The mesolimbic dopamine system: from motivation to action. Wiley, Chichester, pp 19-59
15. Rahman S, McBride WJ (2001) D1-D2 dopamine receptor interaction within the nucleus accumbens mediates long-loop negative feedback to the ventral tegmental area (VTA). J Neurochem 77:1248-1255

16. Kalivas PW, Nakamura M (1999) Neural systems for behavioral activation and reward. Curr Opin Neurobiol 9:223-227

17. Cardinal RN, Parkinson JA, Hall J, Everitt BJ (2002) Emotion and motivation: the role of the amygdala, ventral striatum, and prefrontal cortex. Neurosci Biobehav Rev 26:321-352

18. Grace AA, Bunney BS, Moore H, Todd CL (1997) Dopamine-cell depolarization block as a model for the therapeutic actions of antipsychotic drugs. Trends Neurosci 20:31-37

19. Grace AA (2000) Gating of information flow within the limbic system and the pathophysiology of schizophrenia. Brain Res Brain Res Rev 31:330-341

20. Jellinger KA, Bancher C (1998) Senile dementia with tangles (tangle predominant form of senile dementia). Brain Pathol 8:367-376

21. Mirra SS, Heyman A, McKeel D, Sumi SM, Crain BJ, Brownlee LM, Vogel FS, Hughes JP, van Belle G, Berg L (1991) The Consortium to Establish a Registry for Alzheimer's Disease (CERAD). Part II. Standardization of the neuropathologic assessment of Alzheimer's disease. Neurology 41:479-486

22. McKeith IG, Dickson DW, Lowe J, Emre M, O'Brien JT, Feldman H, Cummings J, Duda JE, Lippa C, Perry EK, Aarsland D, Arai H, Ballard CG, Boeve B, Burn DJ, Costa D, Del Ser T, Dubois B, Galasko D, Gauthier S, Goetz CG, GomezTortosa E, Halliday G, Hansen LA, Hardy J, Iwatsubo T, Kalaria RN, Kaufer D, Kenny RA, Korczyn A, et al (2005) Diagnosis and management of dementia with Lewy bodies. Third report of the DLB consortium. Neurology 65:1863-18725

23. Aoki N, Higashi S, Kawakami I, Kobayashi Z, Hosokawa M, Katsuse O, Togo T, Hirayasu Y, Akiyama H (2012) Localization of fused in sarcoma (FUS) protein to the post-synaptic density in the brain. Acta Neuropathol 124:383-394

24. Sun A, Nguyen XV, Bing G (2002) Comparative analysis of an improved thioflavin-s stain, Gallyas silver stain, and immunohistochemistry for neurofibrillary tangle demonstration on the same Sections. J Histochem Cytochem 50:463-472

25. Arai T, Ikeda K, Akiyama H, Nonaka T, Hasegawa M, Ishiguro K, Iritani S, Tsuchiya K, Iseki E, Yagishita S, Oda T, Mochizuki A (2004) Identification of amino-terminally cleaved tau fragments that distinguish progressive supranuclear palsy from corticobasal degeneration. Ann Neurol 55:72-79

26. Yamada M, Itoh Y, Sodeyama N, Suematsu N, Otomo E, Matsushita M, Mizusawa H (2001) Senile dementia of the neurofibrillary tangle type: a comparison with Alzheimer's disease. Dement Geriatr Cogn Disord 12:117-126

27. Bancher C, Jellinger KA (1994) Neurofibrillary tangle pre- dominant form of senile dementia of Alzheimer type: a rare subtype in very old subjects. Acta Neuropathol 88:565-570

28. Iseki E, Tsunoda S, Suzuki K, Takayama N, Akatsu H, Yamamoto T, Kosaka K (2002) Regional quantitative analysis of NFT in brains of non-demented elderly persons: comparisons with findings in brains of late-onset Alzheimer's disease and limbic NFT dementia. Neuropathology 22(1):34-39

29. Saito Y, Ruberu NN, Sawabe M, Arai T, Tanaka N, Kakuta Y, Yamanouchi H, Murayama S (2004) Staging of argyrophilic grains: an age-associated tauopathy. J Neuropathol Exp Neurol 63:911-918

30. Ikeda K, Akiyama H, Arai T, Sahara N, Mori H, Usami M, Sakata M, Mizutani T, Wakabayashi K, Takahashi H (1997) A subset of senile dementia with high incidence of the apolipoprotein E epsilon2 allele. Ann Neurol 41:693-695

31. Santa-Maria I, Haggiagi A, Liu X, Wasserscheid J, Nelson PT, Dewar K, Clark LN, Crary JF (2012) The MAPT H1 haplotype is associated with tanglepredominant dementia. Acta Neuropathol 124:693-704

32. Janocko NJ, Brodersen KA, Soto-Ortolaza Al, Ross OA, Liesinger AM, Duara R, Graff-Radford NR, Dickson DW, Murray ME (2012) Neuropathologically defined subtypes of Alzheimer's disease differ significantly from neurofibrillary tangle-predominant dementia. Acta Neuropathol 124:681-692

33. Oyanagi K, Takahashi H, Wakabayashi K, Ikuta F (1987) Selective involvement of large neurons in the neostriatum of Alzheimer's disease and senile dementia: a morphometric investigation. Brain Res 411:205-211

34. Oyanagi K, Takahashi H, Wakabayashi K, Ikuta F (1991) Large neurons in the neostriatum in Alzheimer's disease and progressive supranuclear palsy. Brain Res 544:221-226

35. Hasegawa M, Watanabe S, Kondo H, Akiyama H, Mann DM, Saito Y, Murayama (2014) 3R and 4R tau isoforms in paired helical filaments in Alzheimer's disease. Acta Neuropathol 127:303-305

36. Oyanagi K, Makifuchi T, Ohtoh T, Chen KM, Gajdusek DC, Chase TN, Ikuta F (1994) The neostriatum and nucleus accumbens in parkinsonism-dementia 
complex of Guam: a pathological comparison with Alzheimer's disease and progressive supranuclear palsy. Acta Neuropathol 88:122-128

37. Selden N, Mesulam MM, Geula C (1994) Human striatum: the distribution of neurofibrillary tangles in Alzheimer's disease. Brain Res 648:327-331

38. Kim Y, Spruston N (2012) Target-specific output patterns are predicted by the distribution of regular-spiking and bursting pyramidal neurons in the subiculum. Hippocampus 22:693-706

39. Kelley AE, Domesick VB (1982) The distribution of the projection from the hippocampal formation to the nucleus accumbens in the rat: an anterograde- and retrograde-horseradish peroxidase study. Neuroscience 7:2321-2335

40. Weinberger DR, Lipska BK (1995) Cortical maldevelopment, anti-psychotic drugs, and schizophrenia: a search for common ground. Schizophr Res 16:87-110

41. O'Donnell P, Grace AA (1998) Dysfunctions in multiple interrelated systems as the neurobiological bases of schizophrenic symptom clusters. Schizophr Bull 24:267-283

42. Laruelle M (2000) The role of endogenous sensitization in the pathophysiology of schizophrenia: implications from recent brain imaging studies. Brain Res Brain Res Rev 31:371-384

43. Lewis DA, Levitt P (2002) Schizophrenia as a disorder of neurodevelopment. Annu Rev Neurosci 25:409-432

44. Braak H, Zetterberg H, del Tredici K, Blennow K (2013) Intraneuronal tau aggregation precedes diffuse plaque deposition, but amyloid- $\beta$ changes occur before increases of tau in cerebrospinal fluid. Acta Neuropathol 126:631-641

45. Farber NB, Rubin EH, Newcomer JW, Kinscherf DA, Miller JP, Morris JC, Olney JW, McKeel DW Jr (2000) Increased neocortical neurofibrillary tangle density in subjects with Alzheimer disease and psychosis. Arch Gen Psychiatry 57:1165-1173

46. Togo T, Isojima D, Akatsu H, Suzuki K, Uchikado H, Katsuse O, Iseki E, Kosaka K, Hirayasu Y (2005) Clinical features of argyrophilic grain disease: a retrospective survey of cases with neuropsychiatric symptoms. Am J Geriatr Psychiatry 13:1083-1091

47. Holt DJ, Graybiel AM, Saper CB (1997) Neurochemical architecture of the human striatum. J Comp Neurol 384:1-25

doi:10.1186/2051-5960-2-40

Cite this article as: Kawakami et al:: Tau accumulation in the nucleus accumbens in tangle-predominant dementia. Acta Neuropathologica Communications 2014 2:40

\section{Submit your next manuscript to BioMed Central and take full advantage of:}

- Convenient online submission

- Thorough peer review

- No space constraints or color figure charges

- Immediate publication on acceptance

- Inclusion in PubMed, CAS, Scopus and Google Scholar

- Research which is freely available for redistribution 